\title{
Lemierre Syndrome: A Meta-analysis
}

\author{
Mitchell R. Gore ${ }^{10}$ \\ ${ }^{1}$ Department of Otolaryngology, State University of New York \\ Upstate Medical University, Syracuse, New York, United States \\ Int Arch Otorhinolaryngol 2020;24(3):e379-e385.
}

\begin{abstract}
Address for correspondence Mitchell R. Gore, MD, PhD, Department of Otolaryngology, State University of New York Upstate Medical University, Physicians Office Building North, Suite 4P, 4900 Borad Road, Syracuse, NY 13215, United States (e-mail: mgoremdphd@gmail.com).
\end{abstract}

\begin{abstract}
Keywords

- sepsis

- thrombosis

- fusobacterium

Introduction Lemierre syndrome, or postpharyngitis anaerobic sepsis, is an infrequent but life-threatening infection that often involves thrombosis of the internal jugular vein. The role of anticoagulation in addition to antibiotics and surgical treatment remains uncertain.

Objectives 1) To perform a meta-analysis on outcomes and treatment of Lemierre syndrome; and 2) to evaluate the effect of anticoagulation in Lemierre syndrome on vessel recanalization and on mortality.

Data Synthesis A Pubmed database search was conducted using the keywords Lemierre syndrome. A total of 427 studies were identified and reviewed. Data were extracted on patient demographics, treatment type including use and type of anticoagulation, type of antibiotics, presence and location of vessel thrombosis, presence of cranial neuropathies, recanalization of thrombosed vessels on follow-up imaging, organisms isolated on wound or blood cultures, and mortality. The primary outcome variables examined were the effect of anticoagulation on vessel recanalization and mortality. After the review, 359 studies totaling 394 patients between 1990 and 2017 had partial or complete data that could be analyzed. In total, 50 patients had sufficient data on the effect of anticoagulation on vessel recanalization, and 194 had sufficient data on the effect of anticoagulation on mortality. The odds ratio for anticoagulation and vessel recanalization was 1.6 (95\% confidence interval $=0.3$ to $9.4 ; p=0.6)$. The odds ratio for anticoagulation and death was 0.6 ( $95 \%$ confidence interval $=0.1$ to $2.9 ; p=0.5$ ).

Conclusion The present meta-analysis did not demonstrate a statistically significant effect on vessel recanalization or mortality for patients treated with anticoagulation versus patients not anticoagulated in the Lemierre syndrome literature.
\end{abstract}

\section{Introduction}

Pharyngitis leading to sepsis was reported by Schottmuller ${ }^{1}$ in 1918, and in 1936 Andre Lemierre reported on a series of 20 similar cases of postpharyngitis anaerobic sepsis. ${ }^{2}$ Only two of the 20 patients survived, and the syndrome, classically comprised of pharyngeal infection, metastatic septic emboli, internal jugular vein thrombosis, and the presence of the anaerobic bacterium Fusobacterium necrophorum, was eventually named after Lemierre. Lemierre syndrome is an uncommon disease in the postantibiotic era, but can carry a high mortality rate if not recognized and treated aggressively. ${ }^{3}$ The most commonly associated organism, F. necrophorum, is a gram-negative, anaerobic rod-shaped bacterium that is part of the normal oral flora, but multiple other bacteria have been implicated in Lemierre syndrome, such as streptococcal species, Eikenella corrodens, and Staphylococcus aureus. ${ }^{4}$ The venous thrombosis seen in Lemierre syndrome is likely the result of endothelial dysfunction caused by inflammatory factors from the local infection. -Fig. 1 shows axial magnetic resonance imaging received

August 23, 2017

accepted

October 20, 2019

published online

April 24, 2020
DOI https://doi.org/

10.1055/s-0039-3402433. ISSN $1809-9777$.
Copyright (c) 2020 by Thieme Revinter

Publicações Ltda, Rio de Janeiro, Brazil
License terms

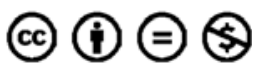




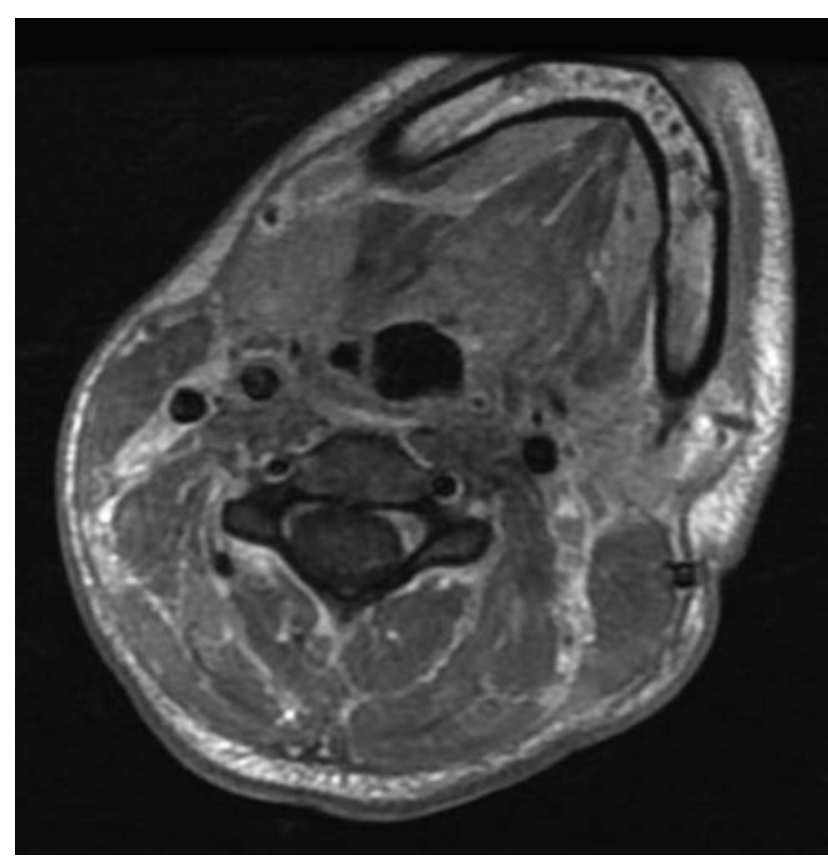

Fig. 1 Axial MRI image showing left jugular vein thrombosis in a patient with Lemierre syndrome.

(MRI) demonstrating left internal jugular vein thrombosis resulting from streptococcal Lemierre syndrome in a young female patient.

Recent studies have examined the role of anticoagulation in the treatment of Lemierre syndrome, ${ }^{5,6}$ but there is no current consensus on the role of anticoagulation and its effect on vessel recanalization in this syndrome. Recent studies have shown equivocal results, with 1 recent retrospective study showing that all patients had improvement in their thrombi by 3 months, with no evident effect on thrombosis outcomes regardless of anticoagulation, ${ }^{5}$ and another showing $\sim 91 \%$ thrombosis improvement and a low complication rate, but with no non-anticoagulated group for comparison. ${ }^{6}$ To our knowledge, there is no meta-analysis available examining the effect of anticoagulation in Lemierre syndrome on vessel recanalization/thrombus resolution, or on mortality. In the present meta-analysis, the aim was to evaluate the effect of anticoagulation on vessel recanalization in patients with Lemierre syndrome with documented follow-up imaging, to examine the effect of anticoagulation on mortality, and to collect data regarding location of thrombosed vessels, antimicrobial treatment type, causative microorganisms isolated on culture, and frequency of associated complications such as cranial nerve palsies.

\section{Review of the Literature}

A thorough literature search of published studies was performed in the Pubmed database using the keywords Lemierre syndrome. Studies not published in English were translated using Google translate (Google/Alphabet, Inc., Mountain View, California, US). Each study was reviewed for relevance and availability of individual patient data. The present study was exempt from Institutional Review Board evaluation, given the use of de-identified retrospective patient data, non-involvement of human subjects, and published literature. The present study was conducted in accordance with the Preferred Reporting Items for Systematic Reviews and Meta-analyses (PRISMA)

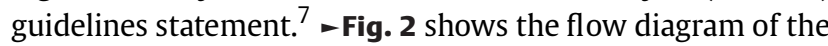
study selection. Patients were included in the meta-analysis if they had evidence of a head-and-neck infection such as pharyngitis/tonsillitis or mastoiditis with at least one other aspect of Lemierre syndrome: metastatic septic emboli or thrombosis of the internal jugular vein, or other documented venous or arterial septic thrombus. Patients with a purely orthopedic or abdominal variant of Lemierre syndrome (extremity infection with thrombosis only of an extremity vessel such as the iliac vein, for example) were excluded, but patients with an abdominal, spinal, or orthopedic thrombosis in conjunction with a head-and-neck infectious source or thrombosed head-and-neck vessel were included. Patients in whom anticoagulation was stopped prior to the attainment of therapeutic levels due to thrombocytopenia or allergic reaction were considered not anticoagulated. Statistical analyses were performed using the Prism (Graphpad Software, San Diego, California, US) software for the Fisher, chi-squared, and number needed to treat (NNT) analyses, and the Medcalc (Medcalc Software, Ostend, Belgium) software for odds ratio (OR) analyses. A two-sided p-value was used and the 95\% confidence interval $(95 \% \mathrm{CI})$ was used for outcome variables.

A total of 676 papers were found and reviewed. The abstracts were screened, and review articles or articles involving only wholly abdominal or orthopedic cases were excluded. A total of 427 studies remained, and these were reviewed for the availability of individual patient data that could be analyzed. A remaining 359 studies (Supplementary Appendix A [online only]) totaling 394 patients had individual patient data that could be examined. Studies were examined for data regarding the location of a thrombosed vessel if present, patient gender, patient age, presence of septic pulmonary or neural tissue emboli, presence of cranial neuropathies, type of antibiotic treatment, microorganisms isolated through blood or tissue cultures, mortality, use of anticoagulation, and resolution of vessel thrombosis on follow-up imaging by ultrasound, computed tomography (CT), or MRI. A total of 50 patients had specific data on resolution or persistence of vessel thrombosis on follow-up imaging and use of anticoagulation, and were included in the vessel recanalization meta-analysis, and 194 patients had data on mortality and use of anticoagulation, and were included in the mortality meta-analysis.

A total of 300 patients had data specifically mentioning the presence or absence of thrombosis. Of these, 283/300 (94.3\%), showed evidence of thrombosis, while $17 / 300$ (5.7\%) showed no evidence of vascular thrombosis. Of the 394 patients included in the study, 120/394 (30.5\%) were treated with antibiotics along with some surgical procedure, while the remaining $274 / 394$ (69.5\%) were treated with antibiotics only. The average age was 28.8 years, and the median age was 23 years. The majority of the patients were between ages 7 and 54, with smaller groupings between ages 2 months to 5 years and 56 to 76 years, with outliers at 80,81 , and 85 years (-Fig. $\mathbf{3}$ ). Of the 344 patients with data reported on 


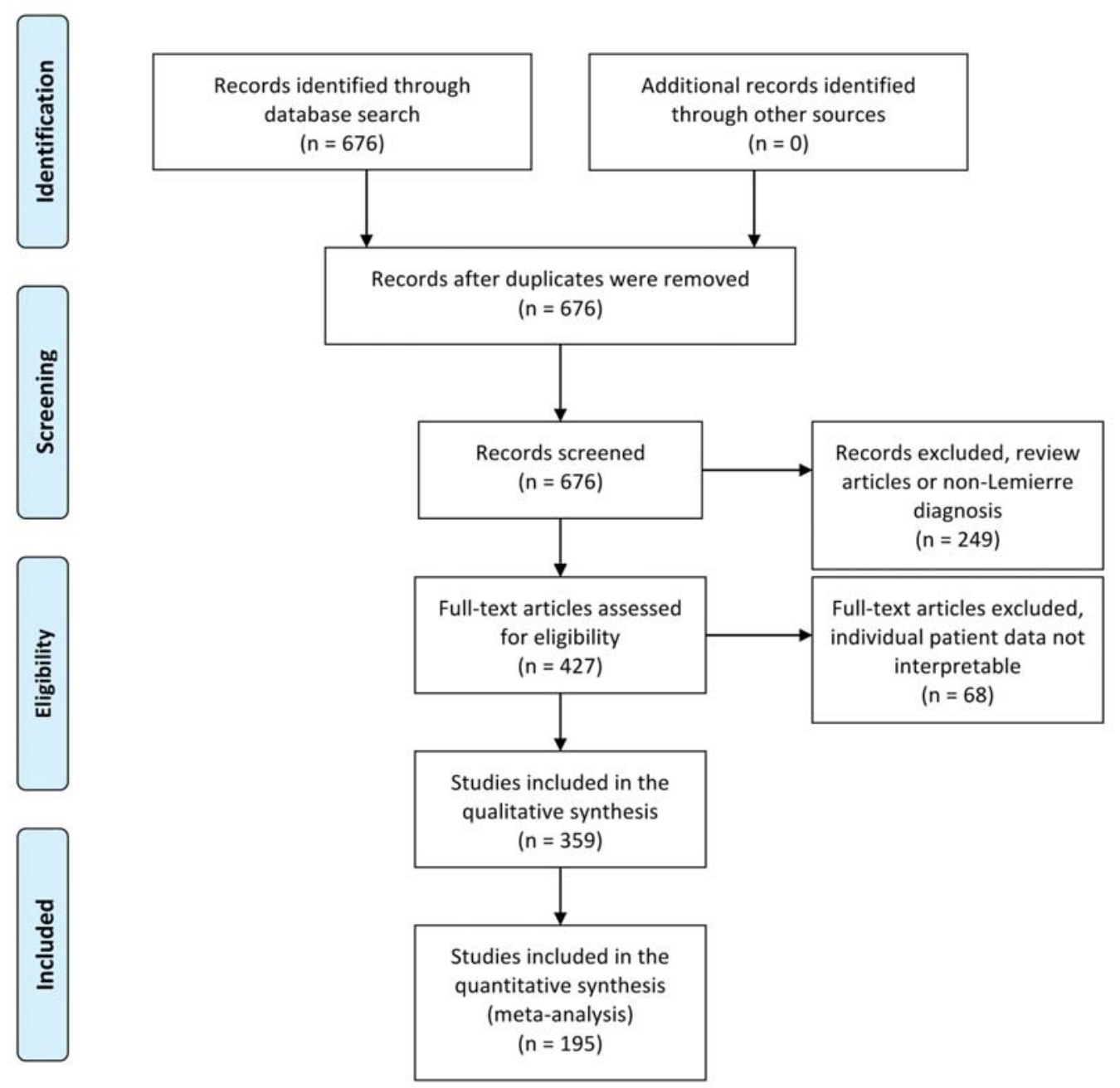

Fig. 2 Flow diagram of the study selection.

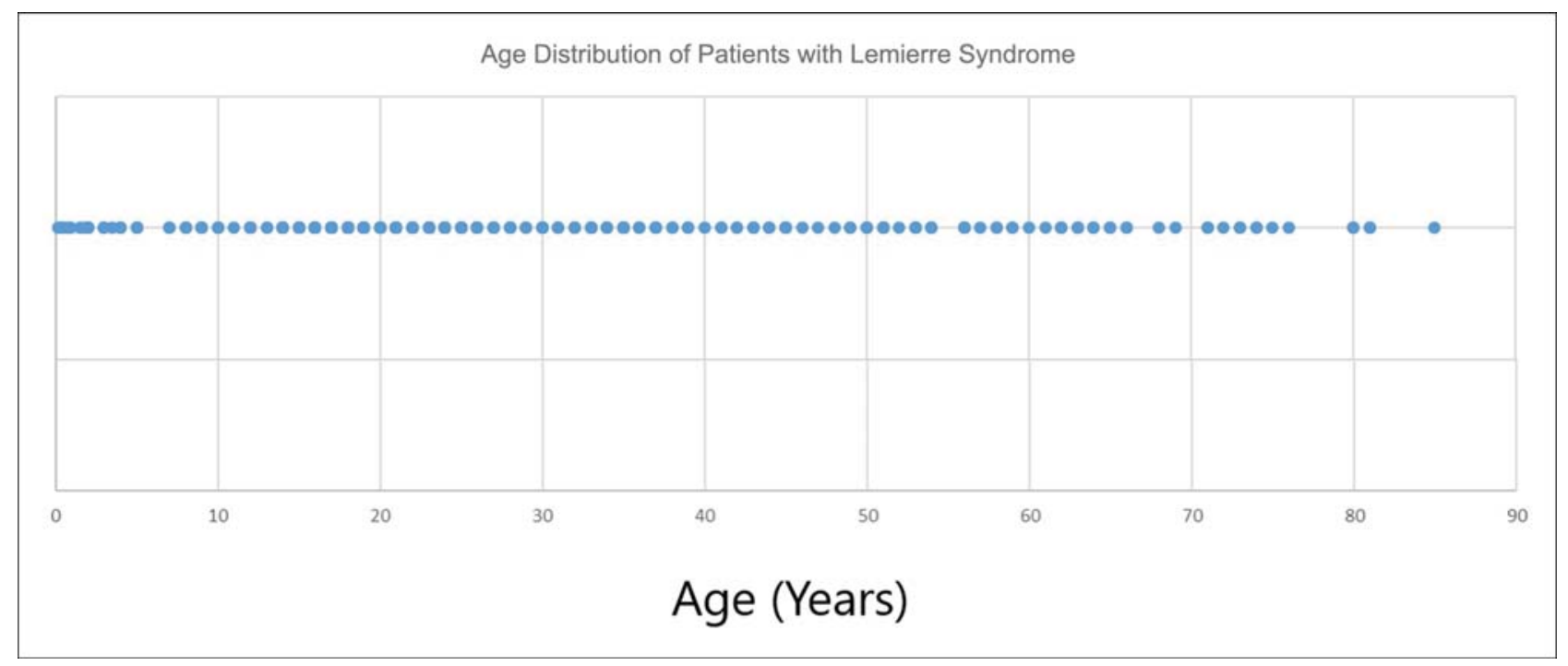

Fig. 3 Age distribution of patients with Lemierre syndrome.

gender, 207/344 (60.2\%) were male, while the remaining $137 / 344$ (39.8\%) were female. There were 16 deaths for a mortality rate of $4.1 \%$. There were 8 patients who developed meningitis, resulting in a meningitis rate of $2.0 \%$. Two patients were pregnant at the time of their diagnosis of Lemierre syndrome. Septic pulmonary emboli were noted in $148 / 394$ patients (37.6\%), while brain emboli were noted in $12 / 394$ patients $(3.0 \%)$. 
The average length of anticoagulation treatment was 81.1 days (range 16-183 days). The average length of antibiotic treatment (intavenous [IV] + oral) was 33.7 days (range 14-60 days). The average time to follow-up imaging was 51.8 days (range 3-365 days). Data collected on the specific vessel or vessels thrombosed, the microbes isolated from blood or tissue cultures, the type of anticoagulation used, and the type of antibiotic therapy used are summarized in - Tables 1-4. Most patients were treated with multiple antibiotics during their treatment, and many patients had thrombosis of multiple vessels. Of the 194 patients for whom use of or lack of use of anticoagulation was specifically noted, 123 (63.4\%) were anticoagulated, while 71 (36.6\%) were not anticoagulated. A total of 23 patients (5.8\%) were noted to have cranial nerve palsies during their clinical course. The cranial nerves involved are summarized in - Table $\mathbf{5}$ (several patients had multiple cranial neuropathies). One patient was also noted to have a Horner syndrome/sympathetic trunk palsy as part of their clinical course. Four patients were noted to have concomitant EpsteinBarr virus (EBV) infection evidenced by positive EBV immunoglobulin $\mathrm{M}(\operatorname{IgM})$, and four patients were noted to have a concomitant malignancy (one laryngeal squamous cell carcinoma in the setting of recurrent respiratory papillomatosis, one serous borderline tumor of the ovary, one lung adenocarcinoma, and one uvular squamous cell carcinoma). Of the 120 patients who underwent a surgical procedure in addition to antibiotic treatment, 3 (2.5\%) underwent endoscopic sinus surgery, $11(9.2 \%)$ underwent mastoidectomy (2 [1.7\%] bilateral), 10 (8.3\%) underwent ligation/excision of the thrombosed internal jugular vein, and $1(0.8 \%)$ underwent ligation/excision of the thrombosed external carotid artery.

After review of the studies, 50 patients were identified with individual data on anticoagulation and recanalization on follow-up imaging. Of the patients treated with anticoagulation, 26 (60.4\%) showed vessel recanalization and 17 (39.5\%) showed persistent vessel thrombosis on follow-up imaging. Of the non-anticoagulated patients, five (71.4\%) showed vessel recanalization and two (28.5\%) showed persistent vessel thrombosis on follow-up imaging. The OR analysis for anticoagulation versus recanalization demonstrated an OR of 1.6 for anticoagulation versus no anticoagulation and vessel recanalization, with a $95 \% \mathrm{CI}$ of 0.3 to 9.4 The two-tailed $p$-value was 0.6 , demonstrating no statistically significant relationship between anticoagulation and vessel recanalization on follow-up imaging. There were 194 patients identified with individual data on anticoagulation and mortality. Of the patients treated with anticoagulation, $120(97.6 \%)$ survived and $3(2.4 \%)$ died, while of the nonanticoagulated patients, 68 (95.8\%) survived and $3(4.2 \%)$ died. The OR for anticoagulation and death was 0.6 , with a $95 \% \mathrm{Cl}$ of 0.1 to 2.9 . The two-tailed $p$-value for anticoagulation and death was 0.5 , demonstrating no statistically significant decrease in mortality with anticoagulation. The number needed to treat (NNT) for anticoagulation and vessel recanalization was 9, while the number needed to treat for anticoagulation and mortality was 55. - Table 6 summarizes the OR ratio analysis for anticoagulation and vessel recanalization and anticoagulation and mortality.
Table 1 Thrombosed vessels noted on imaging

\begin{tabular}{|c|c|}
\hline Thrombosed vessel & $\begin{array}{l}\text { Number of } \\
\text { patients }\end{array}$ \\
\hline Internal jugular vein only & 158 \\
\hline Bilateral internal jugular vein & 11 \\
\hline Contralateral internal jugular vein & 1 \\
\hline Internal jugular vein + another vessel & 87 \\
\hline Subclavian & 10 \\
\hline Sigmoid & 36 \\
\hline External jugular vein & 19 \\
\hline Transverse & 8 \\
\hline $\begin{array}{l}\text { Intracerebral infarct } \\
\text { (venous or arterial) }\end{array}$ & 4 \\
\hline Basilic vein & 1 \\
\hline Supraclavicular vein & 1 \\
\hline Anterior jugular vein & 2 \\
\hline Inferior vena cava & 1 \\
\hline Iliac vein & 2 \\
\hline Superior vena cava & 2 \\
\hline $\begin{array}{l}\text { Multiple vessels not involving the } \\
\text { internal jugular vein }\end{array}$ & 5 \\
\hline Femoral artery & 1 \\
\hline Innominate vein & 1 \\
\hline Facial vein & 7 \\
\hline Thyroid vein & 1 \\
\hline Axillary vein & 4 \\
\hline Splenic vein & 1 \\
\hline Superior mesenteric vein & 1 \\
\hline Anterior cerebral artery & 1 \\
\hline Middle cerebral artery & 1 \\
\hline Maxillary vein & 1 \\
\hline Common femoral vein & 1 \\
\hline $\begin{array}{l}\text { Superior ophthalmic vein or } \\
\text { ophthalmic vein }\end{array}$ & 9 \\
\hline Unilateral cavernous sinus & 20 \\
\hline Bilateral cavernous sinus & 7 \\
\hline Vertebral artery & 2 \\
\hline External carotid artery & 2 \\
\hline Internal carotid artery & 10 \\
\hline Bilateral internal carotid artery & 1 \\
\hline Common carotid artery & 1 \\
\hline Vertebral vein & 2 \\
\hline $\begin{array}{l}\text { Peritonsillar vein/pharyngeal } \\
\text { plexus/pterygoid plexus }\end{array}$ & 3 \\
\hline Brachiocephalic vein & 5 \\
\hline Superior sagittal sinus & 1 \\
\hline Straight sinus & 1 \\
\hline Retromandibular vein & 5 \\
\hline
\end{tabular}


Table 2 Organisms isolated by culture

\begin{tabular}{|c|c|c|}
\hline Culture result & $\begin{array}{l}\text { Number of } \\
\text { patients }\end{array}$ & Percentage \\
\hline Fusobacterium necrophorum & 131 & $33.2 \%$ \\
\hline Fusobacterium species & 20 & $5.1 \%$ \\
\hline Negative culture & 29 & $7.4 \%$ \\
\hline $\begin{array}{l}\text { Spirocheta/Treponema } \\
\text { (Plaut-Vincent angina) }\end{array}$ & 1 & $0.3 \%$ \\
\hline Multiple organisms & 27 & $6.9 \%$ \\
\hline $\begin{array}{l}\text { Fusobacterium species + } \\
\text { another organism }\end{array}$ & 13 & $3.3 \%$ \\
\hline Bacteroides melaninogenicus & 1 & $0.3 \%$ \\
\hline Prevotella bivia & 2 & $0.5 \%$ \\
\hline $\begin{array}{l}\text { Streptococcus intermedius/ } \\
\text { Streptocossus anginosus/ } \\
\text { Streptococcus milleri }\end{array}$ & 5 & $1.3 \%$ \\
\hline Streptococcus pneumoniae & 4 & $1.0 \%$ \\
\hline Streptococcus viridans & 3 & $0.8 \%$ \\
\hline Streptococcus constellatus & 6 & $1.5 \%$ \\
\hline Fusobacterium nucleatum & 9 & $2.3 \%$ \\
\hline Haemophilus influenza & 2 & $0.5 \%$ \\
\hline Proteus species & 1 & $0.3 \%$ \\
\hline Klebsiella oxytoca & 1 & $0.3 \%$ \\
\hline Corynebacterium species & 1 & $0.3 \%$ \\
\hline Bacteroides fragilis & 4 & $1.0 \%$ \\
\hline Streptococcal species & 4 & $1.0 \%$ \\
\hline Porphyromonas asaccharolytica & 3 & $0.8 \%$ \\
\hline Methicillin-sensitive S. aureus & 6 & $1.5 \%$ \\
\hline Methicillin-resistant S. aureus & 16 & $4.1 \%$ \\
\hline Escherichia coli & 1 & $0.3 \%$ \\
\hline Lactobacillus species & 1 & $0.3 \%$ \\
\hline $\begin{array}{l}\text { Coagulase-negative } \\
\text { Staphylococcus }\end{array}$ & 4 & $1.0 \%$ \\
\hline S. aureus unspecified & 6 & $1.5 \%$ \\
\hline $\begin{array}{l}\text { Arcanobacterium/ } \\
\text { Arcanobacterium haemolyticum }\end{array}$ & 4 & $1.0 \%$ \\
\hline Proteus mirabilis & 1 & $0.3 \%$ \\
\hline Peptostreptococcus species & 3 & $0.8 \%$ \\
\hline Streptococcus pyogenes & 4 & $1.0 \%$ \\
\hline Slackia exigua & 1 & $0.3 \%$ \\
\hline Proprionobacterium species & 1 & $0.3 \%$ \\
\hline Mycoplasma pneumoniae & 1 & $0.3 \%$ \\
\hline Fusobacterium varium & 3 & $0.8 \%$ \\
\hline Klebsiella pneumoniae & 9 & $2.3 \%$ \\
\hline Eikenella corrodens & 1 & $0.3 \%$ \\
\hline Peptostreptococcus anaerobius & 2 & $0.5 \%$ \\
\hline Leptotrichia buccalis & 1 & $0.3 \%$ \\
\hline Streptococcus salivarius & 1 & $0.3 \%$ \\
\hline Prevotella intermedia & 2 & $0.5 \%$ \\
\hline Prevotella species & 1 & $0.3 \%$ \\
\hline
\end{tabular}

(Continued)
Table 2 (Continued)

\begin{tabular}{|l|l|l|}
\hline Culture result & $\begin{array}{l}\text { Number of } \\
\text { patients }\end{array}$ & Percentage \\
\hline Streptococcus sanguinis & 1 & $0.3 \%$ \\
\hline Group A strep & 1 & $0.3 \%$ \\
\hline Group C strep & 6 & $1.5 \%$ \\
\hline Group G strep & 2 & $0.5 \%$ \\
\hline Group B strep & 1 & $0.3 \%$ \\
\hline Pseudomonas aeruginosa & 1 & $0.3 \%$ \\
\hline Staphylococcus capitus & 1 & $0.3 \%$ \\
\hline Staphylococcus epidermidis & 2 & $0.5 \%$ \\
\hline Actinomyces myeri & 1 & $0.3 \%$ \\
\hline Parvimonas micra & 1 & $0.3 \%$ \\
\hline Chromobacterium violaceum & 1 & $0.3 \%$ \\
\hline
\end{tabular}

Table 3 Type of anticoagulant used

\begin{tabular}{|l|l|}
\hline Anticoagulant used & Number of patients \\
\hline Total anticoagulation & 123 \\
\hline Total no anticoagulation & 71 \\
\hline Heparin/LMWH/unfractionated heparin & 53 \\
\hline Xarelto/Rivaroxaban & 2 \\
\hline Edoxaban/Savaysa & 2 \\
\hline Warfarin/Coumadin & 29 \\
\hline Enoxaparin/Lovenox & 19 \\
\hline Tinzaparin/Innohep & 2 \\
\hline Bemiparin & 1 \\
\hline Dabigatran/Pradaxa & 1 \\
\hline Fondaparinux/Arixtra & 4 \\
\hline Danaparoid/Orgaran & 1 \\
\hline Dalteparin/LMWH & 1 \\
\hline Nadroparin/Fraxiparine/LMWH & 1 \\
\hline
\end{tabular}

Abbreviation: LMWH, low molecular weight heparin.

\section{Discussion}

The present study analyzed the diagnosis and management in 394 patients with a diagnosis of Lemierre syndrome reported between 1980 and 2017. A meta-analysis was conducted on 194 of these patients to examine the effect of anticoagulation on mortality, and on 50 patients to examine the effect of anticoagulation on vessel recanalization on follow-up imaging. The OR for anticoagulation and mortality was 0.6 , while the OR for anticoagulation and vessel recanalization was 1.6. Neither relationship was statistically significant, showing no effect on mortality or vessel recanalization with anticoagulation in these subsets of Lemierre syndrome patients. Cupit-Link et $\mathrm{al}^{5}$ retrospectively examined the charts of 18 pediatric and adult patients with Lemierre syndrome. In total, 6 of the patients were anticoagulated for at least 4 weeks (range 6.9-32.9 weeks). They noted that all patients had improvement in their 
Table 4 Type of antibiotic used

\begin{tabular}{|c|c|}
\hline Antibiotic used & Number of patients \\
\hline Imipenem or imipenim cilastatin & 10 \\
\hline Ceftazidime & 3 \\
\hline Cefpodoxime & 1 \\
\hline Cefuroxime & 5 \\
\hline Cefepime & 6 \\
\hline Cefotetan & 1 \\
\hline Cefoperazone & 1 \\
\hline Flucloxacillin & 1 \\
\hline Clindamycin & 63 \\
\hline $\begin{array}{l}\text { Ampicillin/sulbactam or } \\
\text { ticarcillin/clavulanate }\end{array}$ & 23 \\
\hline Metronidazole & 83 \\
\hline Chloramphenicol & 2 \\
\hline Piperacillin/tazobactam & 28 \\
\hline Nafcillin & 1 \\
\hline Rifampin & 1 \\
\hline Penicillin/amoxicillin/ampicillin & 34 \\
\hline Methicillin/flucloxacillin/floxacillin & 4 \\
\hline Dicloxacillin & 2 \\
\hline Gentamicin & 12 \\
\hline Aztreonam & 1 \\
\hline Moxifloxacin & 2 \\
\hline Ofloxacin & 1 \\
\hline Pazufloxacin & 1 \\
\hline Daptomycin & 1 \\
\hline Trimethoprim/sulfamethoxazole & 1 \\
\hline Tigecycline & 1 \\
\hline Linezolid & 4 \\
\hline Ertapenem & 3 \\
\hline Vancomycin & 44 \\
\hline Doxycycline & 1 \\
\hline Azithromycin & 2 \\
\hline Cefotaxime & 10 \\
\hline Levofloxacin & 8 \\
\hline Ciprofloxacin & 8 \\
\hline Roxithromycin & 1 \\
\hline Meropenem & 30 \\
\hline Amikacin & 3 \\
\hline Teicoplanin & 2 \\
\hline Biapenem & 1 \\
\hline Clarithromycin & 6 \\
\hline
\end{tabular}

thrombi by three months, with complete resolution in nine of the nonanticoagulated patients and partial resolution in three of the nonanticoagulated patients, and complete thrombus resolution in two of the anticoagulated patients, and partial resolution in four of the anticoagulated patients. Their data
Table 5 Cranial nerve palsies observed

\begin{tabular}{|l|l|}
\hline Cranial nerve palsy & Number of Patients \\
\hline $\begin{array}{l}\text { Total of patients with } \\
\text { cranial nerve palsy }\end{array}$ & 23 \\
\hline I & 3 \\
\hline II & 0 \\
\hline III & 3 \\
\hline IV & 2 \\
\hline V & 1 \\
\hline VI & 9 \\
\hline VII & 3 \\
\hline VIII & 3 \\
\hline IX & 0 \\
\hline X (recurrent laryngeal nerve) & 2 \\
\hline XI & 0 \\
\hline XII & 5 \\
\hline
\end{tabular}

Table 6 Odds ratio analysis for the effect of anticoagulation treatment on vessel recanalization and anticoagulation treatment on mortality in patients with Lemierre syndrome

\begin{tabular}{|l|l|}
\hline Vessel recanalization & \\
\hline Odds ratio & 1.6 \\
\hline 95\% confidence interval & 0.3 to 9.4 \\
\hline Significance level & $p=0.6$ \\
\hline Number needed to treat & 9 \\
\hline Mortality & \\
\hline Odds ratio & 0.6 \\
\hline 95\% confidence interval & 0.1 to 2.9 \\
\hline Significance level & $p=0.5$ \\
\hline Number needed to treat & 55 \\
\hline
\end{tabular}

suggested that anticoagulation in Lemierre syndrome patients did not appear to affect the rate of thrombus resolution. Rebelo et $\mathrm{al}^{6}$ retrospectively examined 11 pediatric patients with Lemierre syndrome and thrombosis (sigmoid sinus: 6; internal jugular vein: 5). All 11 patients were anticoagulated with low molecular weight heparin (LMWH) for an average duration of 105.8 days. In total, 10 of the 11 patients had partial or complete resolution of the thrombus within a median of 3.4 months. No control non-anticoagulated group was analyzed, but they postulated that the benefit of anticoagulation might outweigh the low risks given their observation of no adverse effects from anticoagulation in their retrospective patient series. Their review of the literature revealed anticoagulant use in $63.7 \%$ of pediatric cases reported since 2002, similar to our observed rate of $63.4 \%$ of patients anticoagulated overall since 1980. Interestingly, they noted one middle cerebral artery (MCA) infarct, four patients with septic pulmonary emboli, three cranial nerve palsies, and two patients with Horner syndrome. Phan et $\mathrm{al}^{8}$ reported a single pediatric Lemierre case treated with enoxaparin with persistent internal jugular 
(IJ) vein thrombosis on three-week follow-up imaging, but proposed that anticoagulation should be considered in highrisk patients in the absence of any contraindication to anticoagulation. In a case treated by the author of the present study noted in - Fig. 1, the Infectious Disease Service recommended against anticoagulation due to the risk of intracranial hemorrhage given the patient's thrombocytopenia and sigmoid sinus involvement, while Vascular Surgery recommended anticoagulation. Ultimately no anticoagulation was given, and the patient had an uneventful clinical course with aggressive IV antibiotic treatment, while an MRI at one year (shown in -Fig. 1) showed persistence of the left internal jugular vein thrombosis. The patient had no long-term sequelae of the disease.

A search of the literature did not reveal any studies that systematically examined the relationship between mortality and anticoagulation, which may be due to the low overall mortality rate ( $4.1 \%$ in the present study) in the postantibiotic era. Interestingly, the use of ligation/excision of the thrombosed internal jugular vein was low, with 10 patients in this series treated with IJ vein ligation/excision reported between 1980 and 2017. No studies could be identified systematically examining the effect of vessel ligation/excision on mortality, but excision of the thrombosed vessel appeared to be more likely in patients who continued to decline clinically despite aggressive medical therapy. ${ }^{9}$

There are several limitations to the present study. The retrospective nature of the meta-analysis makes recall bias and selection bias more likely. The varied geographical locations and causative organisms, as well as the variation in the type and length of therapy of both the antibiotic and, when used, anticoagulation regimens make it more difficult to draw conclusions from the meta-analysis data. Additionally, there was variability in the timing of follow-up imaging, ranging from several days to one year, introducing the possibility that over time there might be a greater rate of vessel recanalization with a longer follow-up time regardless of anticoagulation. The majority of patients in both the anticoagulated and non-anticoagulated groups showed vessel recanalization. The modality of follow-up imaging also varied depending on the study, with some patients followed up using ultrasound, while CT or MRI was used in other patients. Nevertheless, the present study represents the largest retrospective series of Lemierre syndrome patients analyzed, and is the first study to our knowledge to formally compare the rates of vessel recanalization and mortality in anticoagulated and non-anticoagulated patients. Neither group showed a statistically significant advantage for anticoagulation. Given the relatively low incidence of Lemierre syndrome, and the difficulty of assembling prospective, randomized patient groups, and the findings of this meta-analysis, it may be reasonable to treat patients primarily with aggressive empiric and then culture directed antibiotic therapy to cover the most common organisms (Fusobacterium, Staphylococcus, and streptococcal species), with surgical treatment as indicated for coalescent mastoiditis, empyema, severe sinusitis, abscesses etc., reserving anticoagulation for patients with low risk of anticoagulation side effects or who have refractory sequelae of vessel thrombosis despite aggressive antibiotic $+/$ - surgical treatment. A more routine follow-up imaging protocol may be useful in analyzing vessel recanalization, but this may be difficult as many Lemierre patients may be lost to follow-up.

\section{Final Comments}

The present meta-analysis examined 394 patients presented in the literature between 1980 and 2017 with a diagnosis of Lemierre syndrome. A meta-analysis of the effect of anticoagulation on vessel recanalization and mortality failed to show a statistically significant benefit for either outcome. Aggressive IV antibiotic therapy combined with surgical intervention when indicated appeared to be the mainstay of treatment in Lemierre syndrome, with a low mortality rate overall with or without anticoagulation.

\section{Author Contributions}

Mitchell Gore: design, data analysis and interpretation, drafting and final approval of the article.

\section{Conflict of Interests}

The author have no conflict of interests to declare.

\section{References}

1 Schottmuller H. Ueber die Pathogenität anaërober Bazillen. Dtsch Med Wochenschr 1918;44:1440 (in German)

2 Lemierre A. On certain septicaemias due to anaerobic organisms. Lancet 1936;227:701-703

3 Cappanera S, Tiri B, Saraca LM, Francisci D. [Let us not forget the Lemierre's Syndrome]. Recenti Prog Med 2016;107(09):487-491

4 Sinave CP, Hardy GJ, Fardy PW. "The Lemierre's syndrome: suppurative thrombophlebitis of the internal jugular vein secondary to oropharyngeal infection". Medicine (Baltimore): Williams \& Wilkins; 198968 (2): 85-94

5 Cupit-Link MC, Nageswara Rao A, Warad DM, Rodriguez V. Lemierre Syndrome: A Retrospective Study of the Role of Anticoagulation and Thrombosis Outcomes. Acta Haematol 2017;137 (02):59-65

6 Rebelo J, Nayan S, Choong K, Fulford M, Chan A, Sommer DD. To anticoagulate? Controversy in the management of thrombotic complications of head \& neck infections. Int J Pediatr Otorhinolaryngol 2016;88:129-135

7 Moher D, Liberati A, Tetzlaff J, Altman DG; PRISMA Group. Preferred reporting items for systematic reviews and meta-analyses: the PRISMA statement. J Clin Epidemiol 2009;62(10): 1006-1012

8 Phan T, So TY. Use of anticoagulation therapy for jugular vein thrombus in pediatric patients with Lemierre's syndrome. Int J Clin Pharm 2012;34(06):818-821

9 Escher R, Haltmeier S, von Steiger N, et al. Advanced Lemierre syndrome requiring surgery. Infection 2008;36(05):495-496 